\title{
Geometric parametric instability in modulated parabolic graded-index fibers
}

\author{
Carlos Mas Arabí, Alexandre Kudlinski, Arnaud Mussot, and Matteo Conforti \\ Univ. Lille, CNRS, UMR 8523-PhLAM-Physique des Lasers Atomes et Molécules, F-59000 Lille, France \\ matteo.conforti@univ-lille1.fr
}

\begin{abstract}
We show that a periodic modulation of the diameter of a graded-index multimode fiber modifies the intrinsic self-imaging pattern, generating new spectral components in the geometric parametric instability gain spectrum.
\end{abstract}

OCIS codes: (060.4370) Nonlinear optics, fibers; (060.5530) Pulse propagation and temporal solitons; (190.4420) Nonlinear optics, transverse effects in.

Parametric resonance (PR) is a well-known instability process which develops in periodically forced systems. This phenomenon was discovered by Faraday in a vertically vibrating fluid, and observed later in several areas of physics $[1,2]$. In nonlinear fiber optics PR appears as the destabilisation of a continuous wave (CW) deriving from the periodic variation of amplification, dispersion or nonlinearity [3]. This periodic variation acts as an external forcing for the system. The periodic evolution of nonlinearity can also be self-induced in highly multimode graded-index (GRIN) fibers with a parabolic profile of refractive index [4]. Indeed, such fibers exhibit a periodic self-imaging of the injected field pattern due to the interference between the different propagating modes. This is due to the fact that the propagation constants of the modes are equally spaced and they have almost identical group velocity [5]. The periodic evolution of the spatial size of the light pattern induces a periodic evolution of the effective nonlinearity in the propagation direction, leading to the generation of unstable bands [6]. This self-induced phenomenon has been named geometric parametric instability (GPI), and recent experiments showed generation of sidebands which can reach detunings from the pump in the order of hundreds of THz [7,8].

In the present work, we study GPI in a system having an internal and external forcing with different periodicity. We consider a multimode GRIN fiber supporting self-imaging (at the origin of the internal forcing), with an additional modulation of the core diameter (inducing the external forcing). The overall longitudinal evolution of the spatial pattern exhibits two spatial frequencies. This results in the generation of characteristic spectral components which differ form the GPI frequencies of a uniform fiber.

Figure 1(a) shows the evolution of the peak intensity of a gaussian beam injected at the center of a modulated GRIN fiber, obtained from the numerical solution of the 3+1D generalized nonlinear Schrödinger equation (GNLSE) [6] (blue curve). The modulation period $L_{\text {mod }}=700 \mu \mathrm{m}$ is slightly larger than the self-imaging period $\xi=600 \mu \mathrm{m}$ and the modulation depth of 3 microns is around ten percent of the core radius $\rho_{0}=26 \mu \mathrm{m}$. The main feature is the modulation of the self-imaging pattern with a period $L \approx 8 L_{\text {mod }}$, which can be interpreded as a Moiré effect. We have calculated analytically an approxiamation of the beam evolution thanks to a multi-scale approach [9] (red curve in Fig. 1(a)), which agrees remarkably well with the numerical simulations (blue curve in Fig. 1(a)). Figure 1(b) shows the spectrum after propagation in a $3 \mathrm{~cm}$ long fiber. We can observe three clusters of spectral peaks located around 120, 170 and $200 \mathrm{THz}$. In a uniform fiber, the GPI resonances manifest as single peaks centrated at the spectral locations $f_{G P I}= \pm \sqrt{m /\left(2 \pi \beta_{2} \xi\right)}, m=1,2, \ldots[6,7]$. The modulation of the fiber generates additional spectral peaks near the main ones. The knowledge of an analytic approximation of the spatial intensity profile in the fiber, allows us to perform a linear stability analysis, which permits to calculate the parametic instability gain spectrum [9]. By choosing a modulation length which satisfies $p L_{m o d}=q \xi$ ( $p, q$ integers), the spatial pattern is periodic of period $L=p L_{m o d}=q \xi$. The periodicity of the pattern permits to use Floquet theory for the calculation of the GPI spectrum. It turns out that the position of the maxima of the parametric gain is given by the following expression:

$$
\omega_{m_{1}, m_{2}}^{2}=\frac{2}{\beta_{2}}\left(-F_{a v}+\sqrt{F_{a v}^{2}+g\left(m_{1}+\frac{m_{2}}{q}\right)^{2}}\right),
$$

where $g_{0}=2 \Delta / \rho_{0}^{2}$ and $F_{a v}$ is a constant which resumes the average strength of the nonlinear forcing and depends on the fiber parameters, the input spot size and the peak power [9]. The index $m_{1}=0,1, \ldots$ counts the main resonances, 
which correspond to the ones obtained in the uniform case. We can observe the generation of new sidebands around the principal ones counted by the second index $m_{2}$. The frequencies calculated by Eq. (1) are shown as vertical dashed lines in Fig. 1(b), which agree remarkably well with GNLSE simulation. It is interesting to investigate the gain spectrum as a function of the ratio $L_{\text {mod }} / \xi$ between the modulation period and the self-imaging distance. Figure 1(c) shows the GPI gain map as a function of frequency and modulation period, calculated by means of the Floquet technique. The corresponding ratio between periods has been chosen to be commensurate and verifies $L_{\text {mod }}=(n+16) /(n+12) \xi$, where $n$ is an integer in the interval $[0,20]$. The overall period of the spatial pattern is thus given by $L=(n+16) \xi$. A remarkable agreement is found with the unstable frequencies predicted by Eq. (1), which are reported in Fig. 1(c) as black dashed curves. For each principal resonance $m_{1}=1,2,3$, one can notice the generation of additional sideband pairs $m_{2}= \pm 4$, whose frequency separation increases with the modulation period. These additional sidebands pairs appear at index $m_{2}= \pm 4$ because, for the definition of the modulation period used in this example, each value of $n$ generates an overall period $L$ composed of four slow oscillations (the pattern is quasi-periodic of period $\approx L / 4$ ). We have verified that results of Floquet analysis are consistent with direct numerical integration of GNLSE [9].
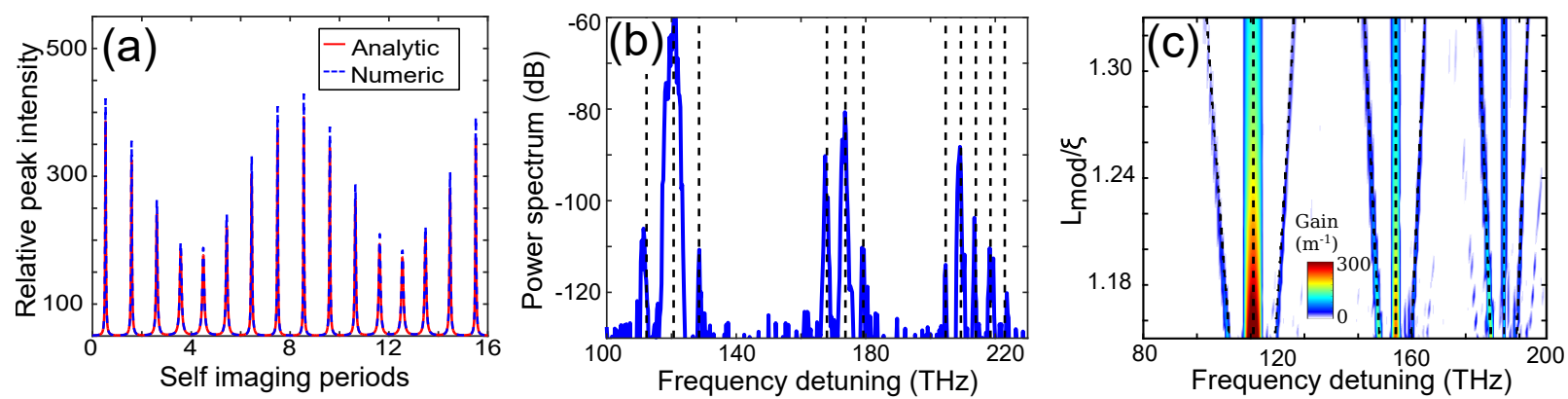

Fig. 1. (a) Evolution of the intensity in the core center normalized to the input one, versus fiber length normalized to the self-imaging period $\xi$, in a fiber with modulation period $L_{\text {mod }}=(8 / 7) \xi$ and amplitude $3 \mu \mathrm{m}$. (b) Spectrum after propagation in a 3-cm long fiber. Vertical dashed lines are theoretical predictions from Eq. (1). (c) Gain map obtained with numerical Floquet analysis as a function of the ratio $L_{\text {mod }} / \xi$. Parameters: $n_{0}=1.47, n_{2}=3.2 \times 10^{-20} \mathrm{~m}^{2} / \mathrm{W}, \rho_{0}=26 \mu \mathrm{m}$, $\Delta=8.8 \times 10^{-3}, \lambda_{0}=1064 \mathrm{~nm}, a_{0}=20 \mu \mathrm{m}, I_{0}=20 \mathrm{GW} / \mathrm{cm}^{2}$ and $\beta_{2}=16.55 \cdot 10^{-27} \mathrm{~s}^{2} / \mathrm{m}$.

To conclude, we have theoretically studied GPI in a graded-index multimode fiber with an axially-modulated core diameter. We have shown that a periodic modulation of the core diameter allows to generate new GPI sidebands. We have developed a theory to predict the frequency of these additional sidebands, which is in excellent agreement with direct numerical simulations of the GNLSE and Floquet stability analysis. Our study contributes to a further understanding of the rich dynamics related to nonlinear waves propagating in multimode fibers.

\section{References}

1. F. Copie, M. Conforti, A. Kudlinski, A. Mussot, and S. Trillo, Phys. Rev. Lett. 116, 143901 (2016).

2. P. Engels, C. Atherton, and M. A. Hoefer, Phys. Rev. Lett. 98, 95301 (2007).

3. A. Mussot, M. Conforti, S. Trillo, F. Copie, and A. Kudlinski, Adv. Opt. Photonics 10, 1 (2018).

4. M. Conforti, C. Mas Arabí, A. Mussot, and A. Kudlinski, Opt. Lett. 42, 4004 (2017).

5. A. Mafi, J. Light. Technol. 30, 2803 (2012).

6. S. Longhi,Opt. Lett. 28, 2363 (2003).

7. K. Krupa, A. Tonello, A. Barthélémy, V. Couderc, B. M. Shalaby, A. Bendahmane, G. Millot, and S. Wabnitz, Phys. Rev. Lett. 116, 183901 (2016).

8. R. Dupiol, A. Bendahmane, K. Krupa, A. Tonello, M. Fabert, B. Kibler, T. Sylvestre, A. Barthélémy, V. Couderc, S. Wabnitz, and G. Millot, Opt. Lett. 42, 1293 (2017)

9. C. Mas Arabí, A. Kudlinski, A. Mussot, and M. Conforti, Phys. Rev. A 97, 23803 (2018). 